\title{
Errorless learning and the cognitive rehabilitation of memory-impaired schizophrenic patients
}

\author{
R. E. O'CARROLL, ${ }^{1}$ H. H. RUSSELL, S. M. LAWRIE AND E. C. JOHNSTONE \\ From the Department of Psychology, University of Stirling; and Department of Psychiatry, University of \\ Edinburgh
}

\begin{abstract}
Background. In recent years, evidence has accumulated that a significant proportion of schizophrenic patients have severe memory impairment, which cannot be attributed to the effects of medication, chronicity or institutionalization. Our group has demonstrated that memory impairment is associated with poor psychosocial outcome and treatment resistance. Work on the classical amnesic syndrome has suggested that memory training is facilitated by adopting an 'errorless learning' approach, where subjects do not experience failure during learning. This is based on the theory that the preserved implicit memory of amnesic patients results in implicitly remembered incorrect responses interfering with target items, in the absence of a functioning explicit memory system to allow differentiation.
\end{abstract}

Method. We compared three groups of subjects, memory-impaired schizophrenic patients, memory unimpaired schizophrenic patients and healthy controls.

Results. An errorless learning approach conferred a significant advantage on the memory-impaired schizophrenic group, bringing their performance up to the level of both control groups. In contrast, adopting a traditional trial and error, or errorful approach resulted in markedly impaired performance in the memory-impaired schizophrenic group only.

Conclusions. We conclude that errorless learning approaches may be worthy of further evaluation in the cognitive rehabilitation of memory-impaired schizophrenic patients.

\section{INTRODUCTION}

Schizophrenia is 'arguably the worst disease affecting mankind' (Editorial Nature, 1988), as it affects young people in their prime and often destroys their ability to have productive independent lives. The advent of neuroleptic medication in 1950s led to marked improvements in treatment, both in controlling the acute episodes and reducing the risk of relapse. However, up to $25 \%$ of patients do not show a substantial therapeutic response to phenothiazines (Davis, 1976). These treatment resistant patients with schizophrenia represent a considerable therapeutic challenge and a burden on health-care systems throughout the world. It is clear that the so-called 'negative

\footnotetext{
${ }^{1}$ Address for correspondence: Dr Ronan E. O'Carroll, Department of Psychology, University of Stirling, Stirling FK9 4LA.
}

features' of schizophrenia are the least likely to respond to medication and are strongly correlated with the degree of cognitive impairment (Owens \& Johnstone, 1980). Furthermore, although symptomatic improvement may occur following introduction of the newer 'atypical' antipsychotics, neuropsychological functioning often remains unaffected (Goldberg et al. 1993). Taken together, negative features and cognitive impairment in schizophrenia represent formidable barriers to successful treatment and rehabilitation.

\section{Memory and schizophrenia}

Traditionally, it was believed that memory impairment was not a central feature of schizophrenia. Bleuler stated categorically that 'memory as such does not suffer in this disease' (Bleuler, 1911) and psychiatric textbooks have continued to promote this view. However, in the 
past few years, there has been a radical reexamination of memory functioning in schizophrenia. Research findings indicate that many schizophrenic patients demonstrate a degree of episodic memory impairment that is indistinguishable from that shown by severely brain damaged patients (McKenna et al. 1990), and this is not an artefact of medication or chronicity, as it is also seen in young medicationnaive patients (Saykin et al. 1991, 1994). Our own work has demonstrated that severity of episodic memory impairment was the only biological (MRI or SPECT) or neuropsychological variable that distinguished treatmentresistant from treatment-responsive schizophrenic patients (Lawrie et al. 1995). Furthermore, Green (1996) has recently reviewed the literature on the functional consequences of neurocognitive deficits in schizophrenia and shown that verbal memory functioning was consistently the best predictor of successful community functioning at follow-up. In many patients, the memory impairment is found in the presence of preserved short-term and implicit memory (Clare et al. 1993; Huron et al. 1995), thus resembling the neuropsychological profile observed in the classic amnesic syndrome (Tamlyn et al. 1992).

\section{Cognitive rehabilitation}

Memory re-training of brain-damaged patients has a long history (Wilson \& Moffat, 1992). It is generally considered that while lost abilities due to brain damage are not truly recoverable, patients can be helped to solve problems via the use of external aids and by learning new strategies for tackling problems that access preserved abilities or skills. A currently influential approach in the field of cognitive rehabilitation is the principle of 'errorless learning', originally developed in animal training experiments. The principle involves the elimination of trail and error approaches to learning. In training, subjects begin with very easy discriminations, do not experience failure, and task difficulty is increased extremely gradually. Sidman \& Stoddard (1967) successfully taught mentally handicapped children to discriminate circles from ellipses using this method. More recently, Baddeley \& Wilson (1994) utilized errorless learning principles in the memory training of amnesic patients and demonstrated that this approach was significantly more effective than the traditional "errorful' trial and error approach. This is thought to be due to the fact that, in the traditional trial and error approach, errors are remembered implicitly and interfere with retrieval of target items (Baddeley \& Wilson, 1994). As stated above, many schizophrenic patients demonstrate an amnesic-like neuropsychological profile with preserved implicit memory functioning and thus, potentially, may derive particular benefit from an errorless learning approach. The only study to date, which has applied errorless learning principles to the cognitive retraining of schizophrenic patients, was reported by Kern et al. (1996). They showed that number of perserverative errors on the Wisconsin Card Sorting Test fell from approximately 40 to $15 \%$ following errorless learning training.

\begin{abstract}
Aim
The experimental hypothesis is that errorless learning will result in significantly better retention than traditional errorful trial and error learning in a sample of memory-impaired schizophrenic patients. Healthy controls and memoryunimpaired schizophrenic patients will show equivalent retention under both methods of learning.
\end{abstract}

\section{METHOD}

\section{Subjects}

Sixty-five patients with a diagnosis of schizophrenia according to DSM-IV criteria were recruited from the Royal Edinburgh and Associated Hospitals. The patients were selected from acute, rehabilitation and out-patient services encompassing a broad range of severity and chronicity. Exclusion criteria included any patients with a history of organic brain disease, head injury, drug or alcohol dependency. None had undergone leucotomy or had received ECT within the previous 12 months. Patients who were taking anti-muscarinic medication were included, but only if their daily dosage did not exceed $20 \mathrm{mg}$ of procyclidine or its equivalent.

All subjects were tested in one individual session, and participated voluntarily without payment. The study received full approval from the local psychiatry and clinical psychology 
ethics committee. During testing, five patients withdrew and one experienced a panic attack. A further five were excluded due to their impaired global cognitive functioning, scoring below 24 out of 30 on the Mini-Mental State Examination (Folstein et al. 1975). Of the remaining 54 patients, 24 were out-patients in moderate to good remission, 23 were in-patients on acute wards, and seven were in-patients from rehabilitation units. Twenty-eight patients were taking procyclidine at a mean daily dosage of $12 \mathrm{mg}$ (range 5-20 mg), one patient was taking benzhexol $2 \mathrm{mg}$ per day and one was taking benzotropine $2 \mathrm{mg}$ per day. Fifty-two patients were taking neuroleptic medication at time of testing (mean chlorpromazine equivalent = $473 \mathrm{mg}$, range 20-1350 $\mathrm{mg}$ per day) (Foster, 1989). (Depixol and Clopixol equivalents were calculated following the guidelines of the manufacturers - Lunbeck.) Eighteen patients were taking atypical antipsychotic medication, six were taking risperidone, mean $5.2 \mathrm{mg}$ per day (range $1-10 \mathrm{mg}$ ), nine were taking clozapine, mean $342 \mathrm{mg}$ per day (range 75-700), one was taking olanzepine $10 \mathrm{mg}$ per day, and one was taking sertindole $20 \mathrm{mg}$ per day. Two patients were drug-free at the time of testing. Chronicity was taken as the time elapsed between the first recorded hospital admission and the date of testing. Mean years chronicity was 10 years, with a range of one month to $34 \cdot 7$ years.

\section{Memory functioning}

Patients were subdivided into three groups according to their performance on memory testing using an abbreviated form of the Rivermead Behavioural Memory Test (RBMT: Wilson et al. 1985). The RBMT was employed as it has been widely used in schizophrenia research (McKenna et al. 1990; Tamlyn et al. 1992; Clare et al. 1993; Duffy \& O’Carroll, 1994; Lawrie et al. 1995) and because it provides an 'ecologically valid' measure of everyday memory functioning that correlates well with observer ratings (Wilson et al. 1989). In the interest of maintaining patient cooperation by keeping testing to a reasonable duration, an abbreviated version of the RBMT was employed. The following subtests were used: face recognition, immediate and delayed story recall and picture recognition. In addition, immediately before the delayed picture recognition task, subjects were asked to recall freely as many of the pictures as they could remember, thus introducing a picture recall as well as a picture recognition subtest. The RBMT subtest raw scores are converted to standardized profile scores $(0,1$ or 2$)$ following Wilson et al. (1985), thus giving a possible total profile score range on this abbreviated version of $0-10$. The classification of memory impairment employed for schizophrenic subjects was scoring below the range of healthy control subjects (see below). Using this method, 20 patients produced scores which fell below the range of healthy controls i.e. less than eight out of 10 , and formed the memory-impaired schizophrenic group (MIS). Twenty-one schizophrenic patients who scored within the normal range on the abbreviated RBMT (i.e. 9-10) formed the memory-unimpaired schizophrenic group (MUS). The remaining 13 schizophrenic patients who scored exactly eight out of 10 on the abbreviated RBMT were designated as undifferentiated with regard to their memory performance, and no further analysis of their results was undertaken.

Twenty people were recruited from the general population as a healthy control group (HC), and all scored between $8-10$ on the abbreviated RBMT. A comparison of the three experimental groups on demographic variables is shown in Table 1. As can be seen, the three groups were extremely well matched.

\section{Measures}

All subjects were administered a battery of neuropsychological tests. The Mini-Mental State Examination (MMSE: Folstein et al. 1975) was used as a brief screening measure of global cognitive functioning. It is generally accepted that a score of 23 or less out of 30 is evidence of significant cognitive impairment (Anthony et al. 1982). Only subjects scoring above this cut-off score were included in the present study. The quick test (Ammons \& Ammons, 1962) was used as a brief measure of verbal IQ. The mental state of the patient was assessed using the Krawiecka Psychiatric Assessment Scale (Krawiecka et al. 1977). Nine variables were rated-depression, anxiety, coherently expressed delusions, hallucinations, incoherence/irrelevance of speech, poverty of speech, flattening of affect, incongruity of affect, psychomotor retardation - each on a five point scale of $0-4$. Observed side-effects 
Table 1. Comparison of the three subject groups on demographic/matching variables

\begin{tabular}{|c|c|c|c|c|c|}
\hline & \multicolumn{2}{|c|}{ Schizophrenic group } & \multirow{2}{*}{$\begin{array}{l}\text { Healthy } \\
\text { controls } \\
(N=20)\end{array}$} & \multirow[b]{2}{*}{$F$} & \multirow[b]{2}{*}{$P$} \\
\hline & $\begin{array}{l}\text { Memory impaired } \\
\quad(N=20)\end{array}$ & $\begin{array}{l}\text { Memory unimpaired } \\
\quad(N=21)\end{array}$ & & & \\
\hline Age & $35 \cdot 6(11 \cdot 6)$ & $36 \cdot 0(12 \cdot 4)$ & $33 \cdot 1(10 \cdot 8)$ & 0.38 & 0.68 \\
\hline Education & $11 \cdot 8(1.4)$ & $13 \cdot 2(2 \cdot 7)$ & $12 \cdot 3(2 \cdot 0)$ & $2 \cdot 16$ & $0 \cdot 12$ \\
\hline IQ & $102 \cdot 8(14 \cdot 1)$ & $108 \cdot 2(13 \cdot 4)$ & $103 \cdot 2(7 \cdot 9)$ & 1.28 & 0.29 \\
\hline Alcohol & $5.5(7.7)$ & $12 \cdot 4(14 \cdot 0)$ & $13 \cdot 5(11 \cdot 4)$ & $2 \cdot 91$ & 0.06 \\
\hline Sex & $11 \mathrm{M}: 9 \mathrm{~F}$ & $15 \mathrm{M}: 6 \mathrm{~F}$ & $12 \mathrm{M}: 8 \mathrm{~F}$ & $\chi^{2}=1 \cdot 26$ & $P=0.53$ \\
\hline Positive symptoms & $3 \cdot 3(2 \cdot 8)$ & $3 \cdot 1(2 \cdot 7)$ & - & $\begin{array}{ll}n & 0.09\end{array}$ & 0.77 \\
\hline Negative symptoms & $0.85(1.35)$ & $0.29(0.57)$ & - & $3 \cdot 11$ & 0.09 \\
\hline Chronicity & $129 \cdot 4(93 \cdot 7)$ & $111.9(92 \cdot 1)$ & - & $0 \cdot 36$ & 0.55 \\
\hline $\mathrm{CPZ}$ & $613(379)$ & $458(308)$ & - & $1 \cdot 19$ & $0 \cdot 25$ \\
\hline Cooperation & $3 \cdot 8(0 \cdot 6)$ & $3 \cdot 9(0 \cdot 4)$ & - & 0.95 & $0 \cdot 16$ \\
\hline
\end{tabular}

IQ, Quick Test IQ; Alcohol, mean units per week; CPZ, mean chlorpromazine equivalents; Chronicity, months since first hospital admission; Positive symptoms, sum of Krawiecka ratings for delusions, hallucinations and incoherence; Negative symptoms, sum of Krawiecka ratings for poverty of speech and flattening of affect.

were also rated on the Krawiecka scale, including tremor, rigidity, dystonic reactions, akathisia, and difficulties with vision on a three point scale of $0-2$. In addition, level of interest/cooperation during the testing session was assessed on a five point scale 1-5 (Shakow, 1981) where a rating of 1 indicates absolute refusal to participate in the study and 5 applies to subjects who demonstrate real effort and show genuine interest in their performance.

\section{Experimental procedure}

Following Baddeley \& Wilson (1994), we used a stem completion task where subjects were given the first two letters of a five letter word and asked to produce the target word to assess recall under two different learning conditions. No two target words had the same initial letter pair. In their study of amnesic patients, Baddeley \& Wilson (1994) used two lists of five words; one list was presented in an errorful learning way another in an errorless way, with the ordering condition counter-balanced across subjects. For the healthy controls, the word list was increased to 10 words to avoid ceiling effects.

\section{Pilot study}

In a short pilot study of this procedure, a group of 10 patients with schizophrenia (all of whom fitted the inclusion criteria mentioned above) comprising six men and four women were tested, mean age $36.9(10.9)$ years, mean quick IQ 104 $(15 \cdot 5)$, mean years of education $12 \cdot 5(2 \cdot 6)$ and mean alcohol consumption 19.6 (23.9) units per week. From this pilot, it was established that due to ceiling effects in both the errorful and errorless conditions, the results were uninformative when Baddeley \& Wilson's protocol (1994) for patients suffering from the classic amnesic syndrome was followed, i.e. five words to be learned in both errorful and errorless learning conditions. The word list was therefore increased to seven words in each condition for the schizophrenic patients, but the 10 word lists employed by Baddeley \& Wilson (1994) were retained for healthy controls in order to avoid ceiling effects.

\section{Errorless learning}

In the errorless learning condition, for a given target word e.g., BRING, a subject is told ' $\mathrm{I}$ am thinking of a five letter word beginning with BR and the word is BRING, please write that down now'. This procedure was repeated for each word on the list and was repeated for the entire list on each of the three learning trials, using the same instructions before proceeding to the test of recall trials.

\section{Errorful learning}

In the errorful learning condition, the subjects were told 'I am thinking of a five letter word beginning with BR, can you guess what it might be?'. Subjects might guess Break, Brook, Brown, Broom, after which the tester would say, 'No, good guesses, but the word is BRING. Please write that down now'. The correct word was 
immediately provided after four incorrect guesses or after $25 \mathrm{~s}$ had elapsed if four guesses had not been forthcoming. In the unlikely event that subjects guessed the target word first time, a substitute word was employed to ensure at least one error in this condition. Again, subjects went through three learning trials each involving the presentation of the entire list, thus, in this condition, subjects were generating guesses, whereas in the errorless learning phase only correct responses were produced.

The first three trials constituted the learning phase. They were followed by nine test trials in three blocks, with a five minute break after blocks one and two. On all test trials, the first two letters of each word were presented and subjects were asked to supply the correct word. The tester would say 'one of the words you wrote down earlier began BR, can you remember it?'. If the subject did not respond, he/she was encouraged to give any appropriate word, even if they felt it was incorrect. During memory testing a wrong answer was corrected by the tester providing the target word. If after $25 \mathrm{~s}$ the subject had not supplied any word, they were deemed to have forgotten it, and marked as having given an incorrect response. All subjects were tested using both errorless and errorful conditions in the same session, employing a counter-balanced design, with each condition separated by a 10 min break.

The quick IQ test was always administered during the $10 \mathrm{~min}$ break between learning conditions and the remaining break times were used to conduct the interview for the Krawiecka Psychiatric Assessment Scale. This design prevented subjects rehearsing the learned items during the breaks and gave some structure and consistency to the conversation across all subjects. The abbreviated RBMT was administered towards the end of the session and the MMSE during the time lapses before delayed recall testing. This ensured that the experimenter was blind to subject grouping while carrying out the errorless and errorful training conditions, as group allocation was not possible until completion of the assessment session.

\section{Statistics}

Between-group differences in demographic/ matching variables were analysed using univariate analysis of variance. Following Baddeley
\& Wilson (1994), the main hypothesis was tested by comparing the performance of the three groups on both errorful and errorless conditions (percentage correct) using repeated measures analysis of variance (three groups and nine test sessions).

\section{RESULTS}

Inspection of Table 1 reveals that there were no significant differences between the three groups on the potential confounding variables of sex, educational background, age, IQ, or alcohol consumption. Furthermore, there were no significant differences between the memory-impaired and memory-unimpaired schizophrenic groups for the clinical variables of chronicity, positive or negative symptom total, mean chlorpromazine drug dosage, or interest/cooperation rating.

The results of the memory testing following errorful learning are presented in Fig. 1. There was a clear effect of group $(F=5 \cdot 1, P=0 \cdot 009)$, time $(F=18 \cdot 7, \quad P=0.000)$ and a significant group by time interaction $(F=1 \cdot 7, P=0 \cdot 044)$. Therefore, the MIS group performed significantly more poorly than the other two control groups when they had been allowed to guess during learning. In contrast, following errorless learning (Fig. 2), when the subjects were prevented from guessing during learning, there was

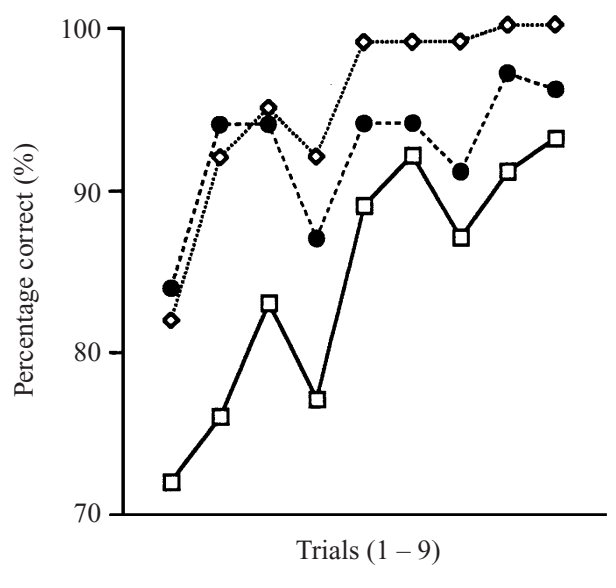

FIG. 1. Memory performance following errorful learning $(\square-\square$, memory impaired schizophrenics $(N=20)$; $\diamond---\diamond$, healthy controls $(N=20)$; --- memory unimpaired schizophrenics $(N=21))$. 


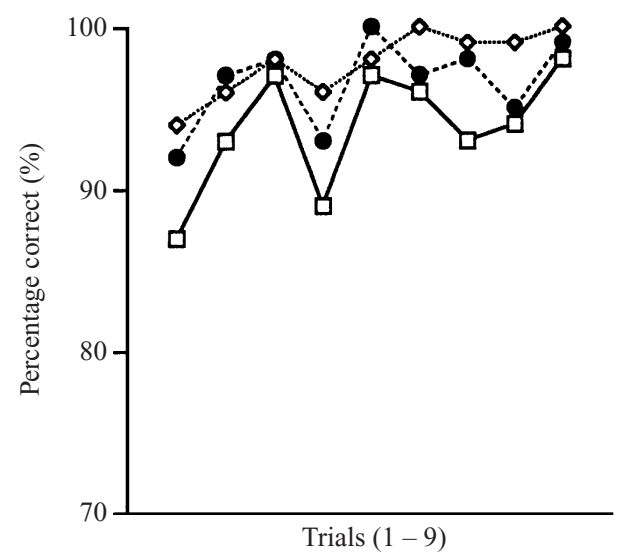

FIG. 2. Memory performance following errorless learning $(\square-\square$, memory impaired schizophrenics $(N=20) ; \diamond---\diamond$, healthy controls $(N=20)$; --phrenics $(N=21))$.

no significant effect of group $(F=1.92, P=$ $0 \cdot 156)$, a significant general effect of time $(F=$ $8 \cdot 79, P=0 \cdot 000)$, but no significant group by time interaction $(F=0.83, P=0.652)$.

Because the two schizophrenic groups were presented with a seven word list, and the healthy control group a ten word list (to avoid ceiling effects), it could be argued that their relative performances are not strictly comparable. Accordingly, a further repeated measures ANOVA was conducted, restricted to the memory-impaired and memory-unimpaired schizophrenic patient groups. For errorful learning, there was a borderline significant effect of group $(F=$ $3.67, P=0.063)$, a clear effect of time $(F=$ $10 \cdot 20, P=0 \cdot 000)$, and a significant group by time interaction $(F=2 \cdot 30, P=0 \cdot 021)$. Following errorless learning, however, there was no effect of group $(F=1.68, P=0.203)$, a significant effect of time $(F=6.57, P=0 \cdot 000)$, but no significant group by time interaction $(F=$ $0 \cdot 52, P=0 \cdot 843)$.

\section{DISCUSSION}

The results of the present investigation indicate that when memory impaired schizophrenic patients are prevented from guessing and making errors during learning, their memory performance is markedly improved. This result cannot be explained away by the possible influence of confounding variables such as differences in age, sex, IQ, educational background, alcohol consumption, medication status, chronicity, symptom profile or general level of cooperation/ motivation, as these were similar between groups. Furthermore, experimenter bias cannot be invoked, as group allocation was made after the errorless/errorful learning experiment had been completed. It is important to note that the results cannot be attributed to ceiling effects, as in both conditions there was scope for improvement as evidenced by a significant time effect, i.e. all three groups improved significantly over the nine trials in both conditions, but only in the errorful condition was there a significant group-by-time interaction.

These results, therefore, support the findings reported by Baddeley \& Wilson 1994), who reported the benefit of errorless learning in patients suffering from the classic amnesic syndrome. As stated in the Introduction, Clare et al. (1993) reported preserved implicit, but markedly impaired, explicit memory functioning in schizophrenia. The results of the present study may, therefore, be attributable to the preserved implicit memory functioning of schizophrenic patients, in the absence of an intact explicit memory system, resulting in incorrect guesses being remembered implicitly and interfering with task performance. Put simply, the memory-impaired schizophrenic patients may have been unable to differentiate between correct and incorrect attempts made during learning. In order to address this issue, further studies need to demonstrate that those subjects who show the greatest benefit from errorless learning have preserved implicit memory functioning. A recent study by Hunkin et al. (1998) addressed this issue with amnesic patients. They showed, however, that improved errorless learning performance was not correlated with preserved implicit memory functioning. They suggested that enhanced performance under errorless learning conditions may be a consequence of some residual explicitly memory functioning. Further work is obviously required in order to characterize the mechanism underlying the errorless learning effect. However, from a practical perspective, consistent benefits have been reported in favour of the errorless learning approach (see also Squires et al. 1997).

An alternative interpretation of the differing performance of the memory-impaired schizo- 
phrenic group across conditions invokes the concept of source monitoring difficulty. Schizophrenic patients (Bentall et al. 1991; Frith et al. 1991; Vinogradov et al. 1997) and patients with frontal lobe lesions (Janowsky et al. 1989) have problems in differentiating self-generated from externally-generated stimuli after a time delay. In the learning phase of the errorful condition, subjects made incorrect guesses and were then given the correct answer by the experimenter i.e. the experimenter was, initially, the source of the correct answer. It is conceivable that in the errorful learning condition, memory-impaired schizophrenic patients had particular difficulty in determining whether the correct answer was a self-generated guess or an experimenter-generated response, as a consequence of source memory problems.

It is ironic that in traditional rehabilitation approaches 'effortful' approaches have generally been advocated. This has been based on the 'levels of processing' approach of Craik \& Lockhart (1972), i.e. that 'working hard' on the material to be remembered leads to better retention. Rehabilitation workers often encourage patients to 'have a guess', in the belief that this will encourage better memory performance. The results of the present study suggest that adopting the traditional approach with memory impaired schizophrenic patients is extremely unhelpful, as making errors during learning leads to confusion during retrieval. This appears to be because patients lack the intact explicit memory system, which is required in order to allow differentiation between correct and incorrect guesses made during learning.

Memory impairment has not been sufficiently recognized in the rehabilitation of schizophrenic patients, because traditionally it has not been considered central to the disorder. It is possible that patients have often failed in rehabilitation settings, not because of apathy, lack of motivation or other negative features that have been attributed to them, but because they have had a genuine and relatively specific impairment of explicit memory functioning. Furthermore, the training methods that have often been employed have utilized and encouraged 'effortful' processing, and 'working' at the material to be remembered. Our results suggest that such approaches with memory impaired schizophrenic patients may have been doomed to failure. Moreover, the experience of repeated failure on the part of the patient may well have led to loss of self-esteem, reduced motivation, and disengagement from the rehabilitation services. We believe that the memory functioning should feature in assessment for rehabilitation, and interventions should be tailored accordingly.

In terms of extrapolating to cognitive rehabilitation in practice, the limitations of the present study are clearly: (a) the relatively short time period over which retention was assessed; and $(b)$ the generalizability of word list learning to 'real life' tasks. In reply, recent studies with amnesic patients have shown that retention following errorless learning can last over a prolonged period of time, and that important everyday activities such as keyboard skills can be trained effectively using an errorless approach (Baddeley \& Wilson, 1994; Wilson et al. 1994). Also, Kern et al. (1996) reported that improvements on the Wisconsin Card Sorting Test, obtained in a chronic schizophrenic sample following errorless learning training, were maintained at 4 week follow-up.

Hogarty \& Flesher (1992) recently stated 'before one embarks on the remediation of cognitive deficits, it would help to know a bit more how a specific deficit or patterns of deficits systematically relates to schizophrenic disability' (p. 53). Evidence is rapidly accumulating that many schizophrenic patients have a marked impairment of secondary memory functioning (McKenna et al. 1990; Saykin et al. 1991; Tamlyn et al. 1992; Clare et al. 1993) and the recent review by Green (1996) indicated that verbal memory functioning was the best predictor of successful reintegration back into the community. We conclude that errorless learning principles are worthy of further evaluation in the cognitive rehabilitation of memory impaired schizophrenic patients.

We would like to thank the subjects who participated in the present study, Norma Brearley for her careful preparation of this manuscript, and the Scottish Office for the grant (ref. no. K/MRS/50/C2526) that made this research possible.

\section{REFERENCES}

Ammons, R. B. \& Ammons, C. H. (1962). Manual for the Quick IQ Test. The Psychological Corporation: New York. 
Anthony, J. C., LeReche, L., Niaz, U., Von Korff, M. R. \& Folstein, M. F. (1982). Limits of the 'Mini-Mental State' as a screening test for dementia and delirium among hospital patients. Psychological Medicine 12, 397-408.

Baddeley, A. \& Wilson, B. A. (1994). When implicit learning fails: amnesia and the problem of error elimination. Neuropsychologia 32, 53-68.

Bentall, R. P., Baker, G. A. \& Havers, S. (1991). Reality monitoring and psychotic hallucinations. British Journal of Clinical Psychology 30, 213-222.

Bleuler, E. (1911). Dementia Praecox, or the Group of Schizophrenias. (Translated 1950 by J. Zinkin.) International Universities Press: New York.

Clare, L., McKenna, P. J., Mortimer, A. M. \& Baddeley, A. D. (1993). Memory in schizophrenia: what is impaired and what is preserved? Neuropsychologia 31, 1225-1241.

Craik, F. I. M. \& Lockhart, R. S. (1972). Levels of processing: a framework for memory research. Journal of Verbal Learning and Verbal Behavior 11, 671-684.

Davis, J. M. (1976). Recent developments in drug treatment of schizophrenia. American Journal of Psychiatry 133, 208-214.

Duffy, L. \& O'Carroll, R. (1994). Memory impairment in schizophrenia - a comparison with that observed in the alcoholic Korsakoff syndrome. Psychological Medicine 24, 155-165.

Editorial (1988). Where next with psychiatric illness? Nature 336, 95-96.

Folstein, M. F., Folstein, S. E. \& McHugh, P. R. (1975). MiniMental State: a practical method for grading the cognitive state of patients for the clinician. Journal of Psychiatric Research 12, 189-198.

Foster, P. (1989). Neuroleptic equivalence. Pharmaceutical Journal 30 (September), 431-432.

Frith, C. D., Leary, J., Cahill, C. \& Johnstone, E. C. (1991). Performance on psychological tests. Demographic and clinical correlates of the results of these tests. British Journal of Psychiatry 159, 26-29.

Goldberg, T. E., Greenberg, R. D., Griffin, S. J., Gold, J. M., Kleinman, J. E., Pickar, D., Schulz, S. C. \& Weinberger, D. R. (1993). The effect of clozapine on cognition and psychiatric symptoms in patients with schizophrenia. British Journal of Psychiatry 162, 43-48.

Green, M. F. (1996). What are the functional consequences of neurocognitive deficits in schizophrenia? American Journal of Psychiatry 153, 321-330.

Hogarty, G. E. \& Flesher, S. (1992). Cognitive remediation in schizophrenia: proceeed ... with caution! Schizophrenia Bulletin 18, 51-57.

Hunkin, N. M., Squires, E. J., Parkin, A. J. \& Tidy, J. A. (1998). Are the benefits of errorless learning dependent on implicit memory? Neuropsychologia 36, 25-36.

Huron, C., Danion, J.-M., Giacomoni, F., Grangé, D., Robert, P. \& Rizzo, L. (1995). Impairment of recognition memory with, but not without, conscious recollection in schizophrenia. American Journal of Psychiatry 152, 1737-1742.

Janowsky, J. S., Shimamura, A. P. \& Squire, L. R. (1989). Source memory impairment in patients with frontal lobe lesions. Neuropsychologia 27, 1043-1056.
Kern, R. S., Wallace, C. J., Hellman, S. G., Womack, L. M. \& Green, M. F. (1996). A training procedure for remediating WCST deficits in chronic psychotic patients: an adaptation of errorless learning principles. Journal of Psychiatric Research 30, 283-294.

Krawiecka, M., Goldberg, D. \& Vaughn, M. (1977). A standardised psychiatric assessment scale for rating psychotic patients. Acta Psychiatrica Scandinavica 55, 299-308.

Lawrie, S. M., Ingle, G. T., Santosh, C. E., Rogers, A. C., Rimmington, J. E., Naidu, K. P., Best, J. J. K., O'Carroll, R. E., Goodwin, G. M., Ebmeier, K. P. \& Johnstone, E. C. (1965). Single photon emission tomography in treatment responsive and treatment resistant schizophrenia. British Journal of Psychiatry 167, 202-210.

McKenna, P. J., Tamlyn, D., Lund, C. E., Mortimer, A. M., Hammond, S. \& Baddeley, A. D. (1990). Amnesic syndrome in schizophrenia. Psychological Medicine 20, 967-972.

Owens, D. G. C. \& Johnstone, E. C. (1980). The disabilities of chronic schizophrenia, their nature and the factors contributing to their development. British Journal of Psychiatry 136, 384-395.

Saykin, A. J., Gur, R. C., Gur, R. E., Mozley, D., Mozley, L. H., Resnick, S. M., Kester, D. B. \& Stafiniak, P. (1991). Neuropsychological function in schizophrenia. Selective impairment in memory and learning. Archives of General Psychiatry 48, 618-624.

Saykin, A. J., Shtasel, D. L., Gur, R. E., Kester, D. B., Mozley, L. H., Stafiniak, P. \& Gur, R. C. (1994). Neuropsychological deficits in neuroleptic naive patients with first-episode schizophrenia. Archives of General Psychiatry 51, 124-131.

Shakow, D. (1981). The place of co-operation in the examination of mental disorder. Journal of Nervous and Mental Disease 169, 127-137.

Sidman, M. \& Stoddard, L. T. (1967). Effectiveness of fading in programming simultaneous form discrimination for retarded children. Journal of Experimental Analysis of Behaviour 10, 3-15.

Squires, E. J., Hunkin, N. M. \& Parkin, A. J. (1997). Errorless learning of novel associations in amnesia. Neuropsychologia 35, 1103-1111.

Tamlyn, D., McKenna, P. J., Mortimer, A. M., Lund, C. E., Hammond, S. \& Baddeley, A. D. (1992). Memory impairment in schizophrenia: its extent, affiliations and neuropsychological character. Psychological Medicine 22, 101-115.

Vinogradov, S., Willis-Shore, J., Poole, J. H., Marten, E., Ober, B. A. \& Shenaut, G. K. (1997). Clinical and neurocognitive aspects of source monitoring errors in schizophrenia. American Journal of Psychiatry 154, 1530-1537.

Wilson, B. A. \& Moffat, N. (1992). Clinical Management of Memory Problems, 2nd edn. Chapman \& Hall: London.

Wilson, B. A., Cockburn, J. \& Baddeley, A. D. (1985). Rivermead Behavioural Memory Test. Thames Valley Test Co.: Titchfield, Fareham.

Wilson, B. A., Baddeley, A. D., Evans, J. \& Shiel, A. (1994). Errorless learning in the rehabilitation of memory-impaired people. Neuropsychological Rehabilitation 4, 307-326.

Wilson, B. A., Cockburn, J. M., Baddeley, A. D. \& Hiorns, R. (1989). The development and validation of a test battery for detecting and monitoring everyday memory problems. Journal of Clinical and Experimental Neuropsychology 11, 855-870. 